\title{
Sugar Beverages and Dietary Sodas Impact on Brain Health: A Mini Literature Review
}

Ibrar Anjum $^{1}$, Syeda S. Jaffery ${ }^{2}$, Muniba Fayyaz ${ }^{3}$, Abdullah Wajid ${ }^{4}$, Armghan H. Ans ${ }^{5}$

1. Internal Medicine, The University of Texas MD Anderson Cancer Center, Houston, USA 2. Department of Research, California Institute of Behavioral Neurosciences \& Psychology, Skokie, USA 3. Department of Internal Medicine, Fatima Memorial Hospital, Lahore, PAK 4. FMH College of Medicine \& Dentistry, Lahore, PAK 5. Department of Cardiology, University of Pennsylvania, Philadelphia, USA

Corresponding author: Armghan H. Ans, ansarmghan@gmail.com

\begin{abstract}
Sugar-sweetened beverages containing caffeine are widely used among humans nowadays and can have negative consequences on the overall health. Our study aims to discuss the effects of these sugar-sweetened beverages (SSB) and how they can impact the health in different ways particularly on the brain. Some of the mechanisms by which soft drinks can exert adverse effects include an increase in glutathione-6dehydrogenase level, increased levels of gamma-aminobutyric acid (GABA), glutamate and dopamine alteration in brain waves on electroencephalography (EEG) eventually leading to stroke and dementia. They can increase the oxidative stress by a decreasing monoamine oxidase and acetylcholine esterase and antioxidants such as glutathione and catalase. The sleep quality and duration of sleep is also significantly affected by their increased consumption. Also, the consumption of sodium benzoate (found in beverages) on impairing memory, motor coordination, affecting reduced glutathione (GSH), increasing the malondialdehyde (MDA) level in the brain and producing attention deficit hyperactivity disorder (ADHD) in children is emphasized. Finally, we will highlight how diet drinks can also be harmful and the maternal consumption of chocolate or soft drinks during pregnancy and postnatal period can be linked to cognitive impairment and child obesity.
\end{abstract}

Received 05/23/2018 Review began 05/30/2018 Review ended 06/04/2018 Published 06/07/2018

(c) Copyright 2018 Anjum et al. This is an open access article distributed under the terms of the Creative Commons Attribution License CC-BY 3.0., which permits unrestricted use, distribution, and reproduction in any medium, provided the original author and source are credited.
Categories: Internal Medicine, Neurology, Miscellaneous

Keywords: effects of soda and dietary consumptions on brain, soft drinks effect on congnitive ability, soda and other drinks role in sleep, caffeine and brain, sugar-sweetened beverages and its effect on mind

\section{Introduction And Background}

In today's advancing society, the use of soda beverages has become necessary if not somewhat unavoidable. Whether the beverage consists of carbonated-caffeine drinks such as cola or diet drinks like diet cola, everyone enjoys the pleasurable and refreshing taste of a fizzy drink. While these fizzy drinks may have their uplifting and thirst-quenching satisfaction, they correspondingly play a role in hampering and impacting the normal physiological functioning of the brain [1]. The main component in carbonated-soft drinks is caffeine which triggers the excitation of the reticular system within the brain. Excess excitation of the system leads to insomnia, psychomotor agitation and headaches [2]. These drinks not only provide a refreshing and pleasurable taste but they also come with significant liability. El-Terras et al. conducted a study on Wistar rats for evaluating chronic soda use to gene expression. The analysis reported significant imbalance and altered gene expression also known as oxidative stress within the brain including decreased levels of monoamine oxidase and acetylcholine esterase enzymes. These rats also exhibited decreased levels of antioxidants including Catalase and Glutathione Reductase [3]. Though many prefer diet drinks to regular carbonated sodas for their decreased health risks, in a study published by European Journal of Clinical Nutrition, aspartame, the main ingredient used in diet drinks, was linked to insomnia, headaches, and seizures and in chronic cases blindness, neurotoxicity and memory loss [4,5]. The reason behind is the breakdown of aspartame into phenylalanine, aspartic acid, and methanol, the main components of aspartame. These breakdown products although play a vital role in the body's growth and development. The excess accumulation of these components due to diet drink intake results in cytotoxic effects [6]. Whether the use of carbonated-caffeine cola or diet cola, artificially flavoured drinks and their increased caloric intake demonstrate and support brain dysfunction, dementia and stroke [7].

\section{Review}

\section{Soda and dietary consumption in today's era}

Sugar-sweetened beverages (SSBs) are the leading sources of added sugars in the diet and are increasing on a global level. Caffeine is the central part of a human's diet and is also one of the main components of many foods and beverages, including coffee, tea, soft drinks, dietary drinks, energy drinks and its related products. According to World Health Organization (WHO) Guideline 2015 on the intake of free sugars, a single can of sugar-sweetened soda contains about the upper limit of the recommended 25-50 grams per day [8]. 


\section{Effect of caffeine on brain}

Caffeine is a stimulant frequently consumed by adults and children. Caffeine consumption between 100 and $400 \mathrm{mg}$ has been associated with an increased risk of nervousness, jitteriness, and fidgetiness. Because of continued brain development involving myelination and pruning processes in children, it is harmful to their health. Owolabi et al. conducted a study on rats by administering caffeine and cannabis. It resulted in increased level in glutathione-6-phosphatase dehydrogenase. Also, glutamate, gamma-aminobutyric acid (GABA), and dopamine were increased [9]. A recent study conducted by Xu and Reichelt on rats observed the effects of caffeinated beverages and sucrose on the brain. The study concluded that both ingredients affect the brain causing anxiety-like changes and altering the level of parvalbumin (a calcium-binding protein) in the hippocampus which acts as the second messenger, organizing microtubules. Its alteration in the brain is noticed in patients with Alzheimer's disease [10]. Pase et al. conducted a prospective cohort study on consumption of artificially sweetened drinks. The study revealed that artificially sweetened drinks are associated with a higher risk of stroke and dementia [11]. Free sugars are also the essential ingredient in drinks and essential cause of dental caries [12]. These include monosaccharides like glucose, fructose and disaccharides like sucrose or table sugar which are added to foods and drinks by the manufacturer, cook or consumer [13]. In a recent survey, $48 \%$ underestimated the content of fruit juices and smoothies on average, whereas the sugar content of carbonated drinks was overestimated by $12 \%$ [14]. Also, Meng et al. conducted a study which showed the effect of drinks like coke and other soft drinks on the brain. They investigated the effects of soft drinks and regular coffee on electroencephalography (EEG) signals under resting state and on the performance of motor imaginary-based brain-computer interface. It was concluded that these drinks affect brain waves in different patterns [15]. Pase et al. contributed new data to this debate using prospective data from the Framingham Offspring Cohort study. They investigated the relationship between recent and long-term consumption of sugar, artificially sweetened beverages and the risks of incident stroke and dementia [16]. Furthermore, a recent study by Lebda et al. was done on rats to understand the effect of aspartame in soft drink on brain function and its association between energy status in the brain, oxidative stress and molecular pathways of apoptosis. It was concluded that it affects rat's brain energy function by inhibiting serum and brain creatinine kinase. It also alters electrolytes by increasing calcium and sodium and decreasing copper, iron, zinc, and potassium. Furthermore, soft drinks can also affect hormonal levels in the blood by increasing thyroid hormone T4 and parathyroid hormones and lowering thyroid hormone $\mathrm{T} 3$ and aldosterone levels [17].

\section{Role of drinks in sleep patterns}

Consumption of soft drinks and other beverages is mostly seen in children and young adults. It affects sleep pattern and its quality especially in students [18]. A study conducted by Marmorstein investigated how caffeinated drinks affect sleep in young adolescents. He found that young adolescents who consume energy drinks and coffee are at unusually high risk of alcohol abuse [19]. Also, a recent study conducted by Chaput et al. illustrated that short sleep duration among children is associated with a longer intake of regular soft drink and earlier bedtimes are associated with regular use of soft drinks and other beverages [20].

\section{Drinks consumption and its effect on children}

Nowadays, children are getting $30 \%$ of sugar intake by soft drinks on a regular basis [21]. Sodium benzoate (SB) is found in many food and beverages. Its increased consumption especially in soft drinks and other beverages in children links to attention deficit-hyperactivity disorder. A study conducted by Khoshnoud et al. investigates the effect of different levels of SB on mice brain. It was concluded that SB significantly impairs memory and motor coordination, reduces glutathione (GSH) level and increases the malondialdehyde (MDA) level in the brain. However, some alteration was observed in the Acetylcholinesterase (AChE) Activity. These findings suggest that short-term consumption of SB can impair memory performance and increase brain oxidative stress in mice [22].

\section{Use of soft and dietary drinks during pregnancy and its impact on offspring and children}

A study was conducted by Kjaergaard et al. in which he concluded the effect of maternal intake of chocolate and soft drink and its long-term consequences for the metabolic phenotype in the offspring if they continue to take high sucrose soft drink supplement diet in postnatal life. These offspring showed signs of obesity despite lowered energy intake associated with alterations in the hypothalamic leptin signalling [23]. Also, a study conducted by Cohen et al. shows sugar consumption, especially from SSBs during pregnancy and childhood, may adversely affect child cognition. However, healthy food and nutrition intake can prevent it and may lead to improvement of cognition [24].

\section{Conclusions}

In conclusion, the overall consumption of soft drinks containing sugar and caffeine is increasing drastically worldwide, especially among the young adults. Whether it is regular or diet soda, they all have been known to produce mechanisms harming the human body. Therefore, this issue should be emphasized widely, and more awareness should be raised to decrease its further use to improve the quality of life thus eliminating its negative consequences. Overall, more research is still required regarding this topic. 


\section{Additional Information \\ Disclosures}

Conflicts of interest: In compliance with the ICMJE uniform disclosure form, all authors declare the following: Payment/services info: All authors have declared that no financial support was received from any organization for the submitted work. Financial relationships: All authors have declared that they have no financial relationships at present or within the previous three years with any organizations that might have an interest in the submitted work. Other relationships: All authors have declared that there are no other relationships or activities that could appear to have influenced the submitted work.

\section{References}

1. Eluwa M, Inyangmme I, Akpantah A, Ekanem T, Ekong M, Asuquo O, Nwakanma A: A comparative study of the effect of diet and soda carbonated drinks on the histology of the cerebellum of adult female albino Wistar rats. Afr Health Sci. 2013, 13:541-545. 10.4314/ahs.v13i3.1

2. Wierzejska R: Caffeine-common ingredient in a diet and its influence on human health (Article in Polish) . Rocz Panstw Zakl Hig. 2012, 63:141-147.

3. El-Terras A, Soliman MM, Alkhedaide A, Attia HF, Alharthy A, Banaja AE: Carbonated soft drinks induce oxidative stress and alter the expression of certain genes in the brains of Wistar rats. Mol Med Rep. 2016, 13:3147-3154. 10.3892/mmr.2016.4903

4. Humphries P, Pretorius E, Naude H: Direct and indirect cellular effects of aspartame on the brain . Eur J Clin Nutr. 2008, 62:451-462. 10.1038/sj.ejcn.1602866

5. Ashok I, Sheeladevi R: Biochemical responses and mitochondrial mediated activation of apoptosis on longterm effect of aspartame in rat brain. Redox Biol. 2014, 2:820-831. 10.1016/j.redox.2014.04.011

6. Ashok I, Sheeladevi R, Wankhar D: Long term effect of aspartame (artificial sweetener) on membrane homeostatic imbalance and histopathology in the rat brain. Free Radic Antioxidants. 2013, 3:42-49. 10.1016/j.fra.2013.09.003

7. Pase MP, Himali JJ, Beiser AS, et al.: Sugar-and artificially sweetened beverages and the risks of incident stroke and dementia: a prospective cohort study. Stroke. 2017, 48:1139-1146. 10.1161/STROKEAHA.116.016027

8. WHO calls on countries to reduce sugars intake among adults and children . (2015). Accessed: March 21, 2018: http://www.who.int/mediacentre/news/releases/2015/sugar-guideline/en/.

9. Owolabi JO, Olatunji SY, Olanrewaju AJ: Caffeine and cannabis effects on vital neurotransmitters and enzymes in the brain tissue of juvenile experimental rats. Ann Neurosci. 2017, 24:65-73. 10.1159/000475895

10. Xu TJ, Reichelt AC: Sucrose or sucrose and caffeine differentially impact memory and anxiety-like behaviours, and alter hippocampal parvalbumin and doublecortin. Neuropharmacology. 2018, 137:24-32. 10.1016/j.neuropharm.2018.04.012

11. Pase MP, Himali JJ, Beiser AS, et al.: Sugar- and artificially sweetened beverages and the risks of incident stroke and dementia: a prospective cohort study. Stroke. 2017, 48:1139-1146. 10.1161/STROKEAHA.116.016027

12. Moynihan PJ, Kelly SAM: Effect on caries of restricting sugars intake: systematic review to inform WHO guidelines. J Dent Res. 2014, 93:8-18. 10.1177/0022034513508954

13. Lobstein T: Reducing consumption of sugar-sweetened beverages to reduce the risk of childhood overweight and obesity. World Health Organisation. 2014, Accessed: 2018: http://www.who.int/elena/titles/commentary/ssbs_childhood_obesity/en/.

14. Gill JM, Sattar N: Fruit juice: just another sugary drink?. Lancet Diabetes Endocrinol. 2014, 2:444-446. 10.1016/S2213-8587(14)70013-0

15. Meng J, Mundahl J, Streitz T, Maile K, Gulachek NS, He J, He B: Effects of soft drinks on resting state EEG and brain-computer interface performance. IEEE Access. 2017, 5:18756-18764. 10.1109/ACCESS.2017.2751069

16. Pase MP, Himali JJ, Beiser AS, et al.: Sugar- and artificially sweetened beverages and the risks of incident stroke and dementia: a prospective cohort study. Stroke. 2017, 48:1139-1146. 10.1161/STROKEAHA.116.016027

17. Lebda MA, Sadek KM, El-Sayed YS: Aspartame and soft drink-mediated neurotoxicity in rats: implication of oxidative stress, apoptotic signaling pathways, electrolytes and hormonal levels. Metab Brain Dis. 2017, 32:1639-1647. 10.1007/s11011-017-0052-y

18. Faris MAE, Jahrami H, Al-Hilali MM, Chehyber NJ, Ali SO, Shahda SD, Obaid RS: Energy drink consumption is associated with reduced sleep quality among college students: a cross-sectional study. Nutr Diet. 2017, 74:268-274. 10.1111/1747-0080.12289

19. Marmorstein NR: Interactions between energy drink consumption and sleep problems: associations with alcohol use among young adolescents. J Caffeine Res. 2017, 7:111-116. 10.1089/jcr.2017.0007

20. Chaput JP, Tremblay MS, Katzmarzyk PT, et al.: Sleep patterns and sugar-sweetened beverage consumption among children from around the world. Public Health Nutr. 2018, 23:1-9. 10.1017/S1368980018000976

21. The National Diet and Nutrition Survey: Results from years 1-4 (combined) of the Rolling Programme (2008/2009 - 2011/12). Public Health England and the Food Standards Agency. 2014, Accessed: 2018: https:/www.gov.uk/government/statistics/national-diet-and-nutrition-survey-results-from-years-1-to-4combined-of-the....

22. Khoshnoud MJ, Siavashpour A, Bakhshizadeh M, Rashedinia M: Effects of sodium benzoate, a commonly used food preservative, on learning, memory, and oxidative stress in brain of mice. J Biochem Mol Toxicol. 2018, 32:e22022. 10.1002/jbt.22022

23. Kjaergaard M, Nilsson C, Secher A, et al.: Differential hypothalamic leptin sensitivity in obese rat offspring exposed to maternal and postnatal intake of chocolate and soft drink. Nutr Diabetes. 2017, 7:242. $10.1038 /$ nutd. 2016.53 


\section{Cureus}

24. Cohen JFW, Rifas-Shiman SL, Young J, Oken E: Associations of prenatal and child sugar intake with child cognition. Am J Prev Med. 2018, 54:727-735. 10.1016/j.amepre.2018.02.020 